\title{
ZNF746/PARIS overexpression induces cellular senescence through FoxO1/p21 axis activation in myoblasts
}

Ju-Hyeon Bae ${ }^{1,2}$, Hyeon-Ju Jeong ${ }^{1,2}$, Hyebeen Kim,2, Young-Eun Leem ${ }^{1,2}$, Dongryeol Ryu (1)', Sang Chul Park' Yun-ll Lee ${ }^{3}$, Sung Chun Cho ${ }^{3}$ and Jong-Sun Kang ${ }^{1,2}$

\begin{abstract}
Various stresses, including oxidative stress, impair the proliferative capacity of muscle stem cells leading to declined muscle regeneration related to aging or muscle diseases. ZNF746 (PARIS) is originally identified as a substrate of E3 ligase Parkin and its accumulation is associated with Parkinson's disease. In this study, we investigated the role of PARIS in myoblast function. PARIS is expressed in myoblasts and decreased during differentiation. PARIS overexpression decreased both proliferation and differentiation of myoblasts without inducing cell death, whereas PARIS depletion enhanced myoblast differentiation. Interestingly, high levels of PARIS in myoblasts or fibroblasts induced cellular senescence with alterations in gene expression associated with p53 signaling, inflammation, and response to oxidative stress. PARIS overexpression in myoblasts starkly enhanced oxidative stress and the treatment of an antioxidant Trolox attenuated the impaired proliferation caused by PARIS overexpression. FoxO1 and p53 proteins are elevated in PARISoverexpressing cells leading to p21 induction and the depletion of FoxO1 or p53 reduced p21 levels induced by PARIS overexpression. Furthermore, both PARIS and FoxO1 were recruited to p21 promoter region and Trolox treatment attenuated FoxO1 recruitment. Taken together, PARIS upregulation causes oxidative stress-related FoxO1 and p53 activation leading to p21 induction and cellular senescence of myoblasts.
\end{abstract}

\section{Introduction}

Skeletal muscle is maintained by stem cells called satellite cells, and upon injury quiescent satellite cells are activated, proliferated, and differentiated to regenerate muscles $^{1-3}$. Various pathological conditions are linked with the decline of muscle mass and functionality ${ }^{3}$. The impaired regenerative capacity associated with muscle aging is linked with the reduced number and function of satellite cells due to failure to retain quiescent state and increased senescence ${ }^{4-6}$. Oxidative stress is thought to be

\footnotetext{
Correspondence: Sung Chun Cho (sccho@dgist.ac.kr) or Jong-Sun Kang (kangj01@skku.edu)

${ }^{1}$ Department of Molecular Cell Biology, Sungkyunkwan University School of Medicine, Suwon 440-746, Republic of Korea

${ }^{2}$ Single Cell Network Research Center, Sungkyunkwan University School of

Medicine, Suwon 440-746, Republic of Korea

Full list of author information is available at the end of the article

Edited by P.G. Mastroberardino
}

a main factor for cellular senescence driven by the accumulation of DNA damage and irreversible cell cycle arrest to accelerate stem cell aging and the progression of degenerative diseases ${ }^{5,7}$. Senescent cells exhibit various characteristics such as large flat morphology, senescenceassociated $\beta$-galactosidase (SA- $\beta$-Gal) activity and upregulation of cyclin-dependent kinase inhibitors $\mathrm{p} 16^{\text {Ink4a }}$ (p16) and $\mathrm{p} 21^{\text {Waf1/Cip1 }}$ (p21) leading to cell cycle arrest $^{4,5,8}$. Despite the well-characterized senescent phenotype markers, molecular regulatory pathways for satellite cell senescence are largely unknown. Deregulation of cell cycle inhibitors appears to be the critical mechanisms underlying senescence ${ }^{4,7,8}$.

Among diverse downstream targets of oxidative stress, FoxO transcription factors are recently proposed to play key roles in induction of cellular senescence ${ }^{9-11}$. FoxO transcription factors are originally identified as the

\section{(c) The Author(s) 2020}

(c) (i) Open Access This article is licensed under a Creative Commons Attribution 4.0 International License, which permits use, sharing, adaptation, distribution and reproduction cc) in any medium or format, as long as you give appropriate credit to the original author(s) and the source, provide a link to the Creative Commons license, and indicate if changes were made. The images or other third party material in this article are included in the article's Creative Commons license, unless indicated otherwise in a credit line to the material. If material is not included in the article's Creative Commons license and your intended use is not permitted by statutory regulation or exceeds the permitted use, you will need to obtain permission directly from the copyright holder. To view a copy of this license, visit http://creativecommons.org/licenses/by/4.0/. 
downstream of insulin/insulin growth factor signaling, which is repressed by protein kinase $\mathrm{B}$ ( $\mathrm{PKB} / \mathrm{AKT}$ ) through $^{12,13}$. Interestingly, forced expression of FoxO1 can induce apoptosis in certain cancer cell types, whereas in other cancer cell types, it can induce G1 cell cycle arrest through upregulation of the cyclin-dependent kinase inhibitor $\mathrm{p} 27^{12,14}$. Moreover, in response to the stimulation of transforming growth factor- $\beta$, FoxO proteins form a complex with activated Smad proteins to induce the expression of $\mathrm{p} 21$, leading to cell cycle arrest ${ }^{15,16}$. Thus, the diverse functions of FoxO proteins are dependent on the cellular contexts likely linked with distinct regulatory proteins.

ZNF746 (PARIS) has been originally identified as a substrate of E3 ligase Parkin and its accumulation has been observed in brains of Parkinson's patients ${ }^{17}$. Although the molecular mechanism of PARIS is not completely understood, PARIS suppresses the transcription of peroxisome proliferator-activated receptor- $\gamma$ coactivator 1- $\alpha$ (PGC-1 $\alpha)$, the master regulator of mitochondria biogenesis, through binding to the insulin response element (IRE), the same consensus sequence for FoxOs in the $P G C-1 \alpha$ promoter region ${ }^{17,18}$. In addition, PARIS is implicated in regulation of invasion and epithelial to mesenchymal transition of lung cancer cells and in promotion of colorectal cancer progression via enhancing c-Myc stability ${ }^{19}$. However, the detailed molecular mechanisms and other targets of PARIS need to be characterized. In this study, we explored the role of PARIS in the control of myoblast function. Forced expression of PARIS in myoblasts suppresses myogenic differentiation, whereas PARIS depletion enhances differentiation. PARIS overexpression elicits reduced proliferation and cellular senescence with p21 upregulation. Consistently, the transcriptome analysis of PARIS overexpression reveals dysregulation of genes related to cytokine signaling and cell cycle inhibition. PARIS overexpression triggers oxidative stress and impaired myoblast proliferation, which is rescued by Trolox treatment. Here we demonstrate FoxO1 and p53 are as targets of PARISinduced oxidative stress leading to p21 expression and cellular senescence. Collectively, our results provide evidence that PARIS is a critical regulator to promote myoblast senescence likely contributing to impaired muscle regeneration.

\section{Results}

PARIS overexpression attenuates myoblast differentiation

To examine the role of PARIS in myoblast function, the expression of PARIS was examined during $\mathrm{C} 2 \mathrm{C} 12$ myoblast differentiation. The expression of PARIS was gradually reduced during myoblast differentiation, whereas the level of PGC- $1 \alpha$ was elevated in myoblast differentiation (Fig. 1a and Supplementary Fig. 1a). Next, control pCMV- or PARIS-overexpressing C2C12 cells were differentiated for 3 days (D3), followed by immunostaining for myosin heavy chain (MHC). C2C12/PARIS cells formed predominantly mononucleated MHCpositive myocytes and only a small proportion of myotubes contained two to five nuclei, whereas $\mathrm{C} 2 \mathrm{C} 12 / \mathrm{pCMV}$ cells formed larger myotubes (Fig. 1b-d). Consistently, the protein expression of myogenic markers, $\mathrm{MHC}$ and Troponin $\mathrm{T}(\mathrm{TnT})$ was significantly decreased in $\mathrm{C} 2 \mathrm{C} 12 /$ PARIS cells, relative to control (Fig. 1e, f). To deplete PARIS, two different small interference RNAs (siRNAs) were tested and siPARIS-1 was used in a further study (Supplementary Fig. 1b). PARIS depletion greatly enhanced myotube formation at D2 compared with the scrambled siRNA-expressing cells (Fig. 1g-i). Moreover, the protein level of MHC and TnT was elevated in PARISdepleted cells compared with the control scrambled siRNA-expressing cells (Fig. 1j, k). Taken together, PARIS inhibits myogenic differentiation.

\section{PARIS overexpression reduced myoblast proliferation}

As the proliferation and differentiation of myoblasts are mutually exclusive, we next asked whether PARIS inhibits myoblast differentiation through the promotion of proliferation by 5 -bromo-2'-deoxyuridine (BrdU) incorporation assay. PARIS overexpression in myoblasts caused a significant reduction in the proportion of BrdU-positive cells compared with control (Fig. 2a, b). Furthermore, cell counting experiments revealed that PARIS-overexpressing cells proliferated slower than control cells (Fig. 2c). Next, we determined whether cell death contributes to decreased differentiation of PARIS-overexpressing cells. Thus, control and PARIS-overexpressing $\mathrm{C} 2 \mathrm{C} 12$ cells were subjected to flow cytometry for Annexin-V-fluorescein isothiocyanate (FITC) and propidium iodide (PI). As a control, $\mathrm{C} 2 \mathrm{C} 12$ cells were treated with hydrogen peroxide and $71 \%$ cells were positive for Annexin- $\mathrm{V}$, whereas control and PARIS-overexpressing $\mathrm{C} 2 \mathrm{C} 12$ cells had $8.2 \%$ and 9.8\%, respectively (Supplementary Fig. 2a). In addition, cleaved Caspase3 levels were not different between control and PARIS-overexpressing cells (Supplementary Fig. 2b). Thus, PARIS suppresses the proliferation of myoblasts without affecting cell death.

\section{PARIS triggers cellular senescence}

As PARIS overexpression affected both myoblast proliferation and differentiation, we examined cellular senescence in PARIS-overexpressing cells. Approximately $12 \%$ of C2C12/PARIS cells were positive for SA- $\beta$-Gal staining with flat morphology, whereas few control cells were SA- $\beta$-Gal-positive (Fig. $2 \mathrm{~d}$, e), suggesting that PARIS triggers cellular senescence. To verify, PARIS expression was assessed in cellular senescence of mouse embryonic fibroblasts (MEFs). Freshly isolated MEFs were passaged 


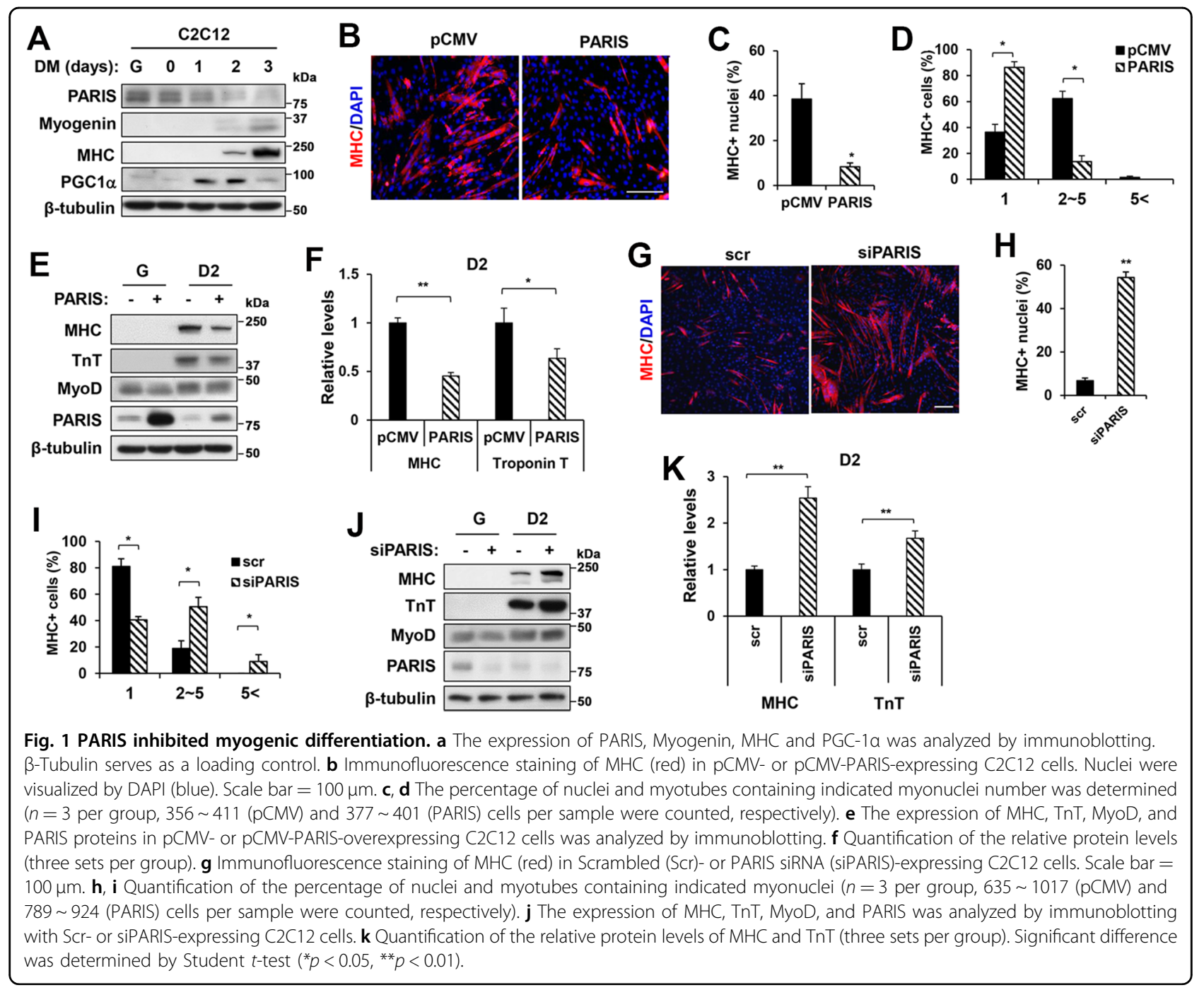

every 2 days and examined the proliferation and senescent phenotypes. At passage 4 (P4), MEFs grew substantially slower and the proliferation was blunted at P7 (Supplementary Fig. 3a). MEFs at P7 showed senescence as indicated by the SA- $\beta$-Gal positivity when compared with unstained MEF at P2 (Supplementary Fig. 3b, c). In addition, the expression of senescence-associated secretory phenotype (SASP) genes (IL6, Igfbp5, Igfbp 7 , and TNF- $\alpha$ ) and p21 was significantly increased in MEFs at P7 (Supplementary Fig. 3d). PARIS transcript levels increased gradually in MEFs with passaging (Supplementary Fig. 3e). The protein levels of PARIS and p21 were greatly elevated in MEFs at P5 and P6 (Supplementary Fig. 3f, g). MEFs at P7 were subjected to immunostaining for PARIS and a proliferation marker Ki67 (Supplementary Fig. 3h, i). The majority of cells were positive for either PARIS or Ki67 and only very low proportion of cells showed double positivity for them. To further examine whether PARIS upregulation is involved in cellular senescence triggered by DNA damage, MEFs were treated with doxorubicin (DOX) for 4 days to induce cellular senescence, as evident by SA- $\beta$-gal staining and the flat morphology (Supplementary Fig. 4a, b). The Dox treatment greatly elevated PARIS protein levels compared with the control treatment (Supplementary Fig. 4c). Similarly, the transcript levels of PARIS and p21 were significantly upregulated in DOX-treated cells, compared with the control (Supplementary Fig. 4d). Thus, the upregulation of PARIS occurs concomitantly with cellular senescence of MEFs.

Next, control or PARIS-transfected C2C12 cells were subjected to immunostaining for $\mathrm{p}-\gamma \mathrm{H} 2 \mathrm{AX}$, a marker for DNA damage, and GL13, a novel chemical analog is highly lipophilic and binds to lipofuscin ${ }^{20}$, associated with cellular senescence. PARIS-transfected $\mathrm{C} 2 \mathrm{C} 12$ cells exhibited elevated $\mathrm{p}-\gamma \mathrm{H} 2 \mathrm{AX}$ foci compared with control cells (Fig. 2f, g). Similar to the SA- $\beta$-Gal staining, PARISoverexpressing cells showed GL13-positive staining, 


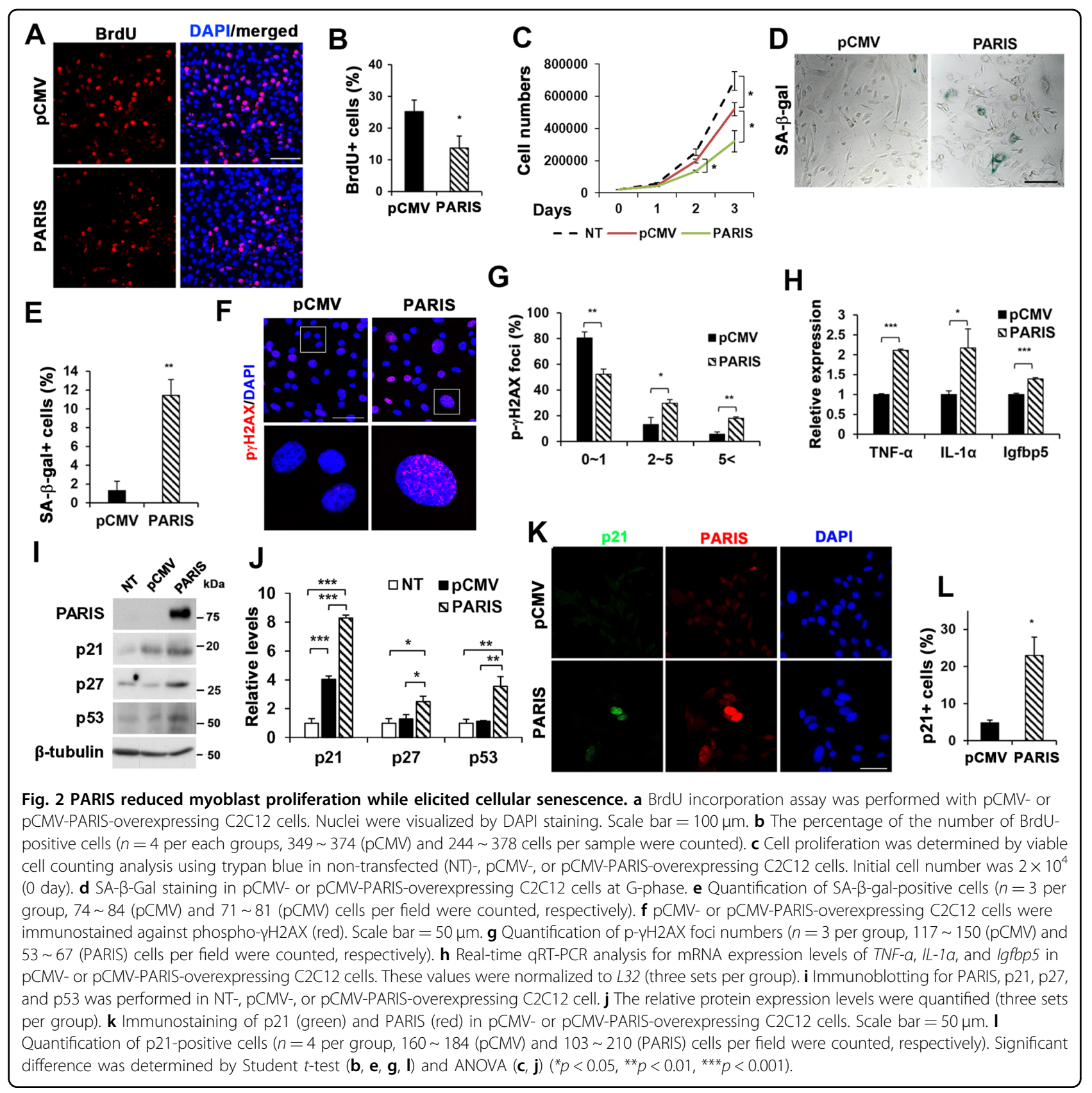

whereas PARIS-negative cells tend to be negative for GL13 (Supplementary Fig. 5). The expression of SASP genes, TNF- $\alpha$, interleukin- $1 \alpha$, and Igfbp 5 , was elevated in PARIS-overexpressing myoblasts compared with control cells (Fig. 2h). In addition, the immunoblotting analysis revealed that PARIS-overexpressing cells had increased levels of cell cycle inhibitors, p21, p27, and p53, compared with pCMV-transfected cells (Fig. 2i, j). In addition, the immunostaining for $\mathrm{p} 21$ and PARIS indicated that about 23\% of PARIS-positive cells were positive for p21 (Fig. 2k, l). Taken together, PARIS upregulation elicits cellular senescence in myoblasts and fibroblasts.
PARIS elevates the expression of genes related to cytokine signaling and cell cycle arrest

Next, we have examined the global gene expression in control or PARIS-overexpressing myoblasts. Genes (1121, 1.5 -fold, $p<0.05)$ were significantly altered in PARISoverexpressing cells (Fig. 3a). The Kyoto Encyclopedia of Genes and Genomes (KEGG) pathway and reactome analysis were performed and the top ten ranked signaling pathways with the cut-off criteria of $p$-value $(<1.6 \mathrm{e}-6)$ and false discovery rate $(<0.05)$ are shown in Fig. $3 \mathrm{~b}$ and Supplementary Fig. 6. The KEGG pathway and Gene Ontology analysis indicated that genes involved in 


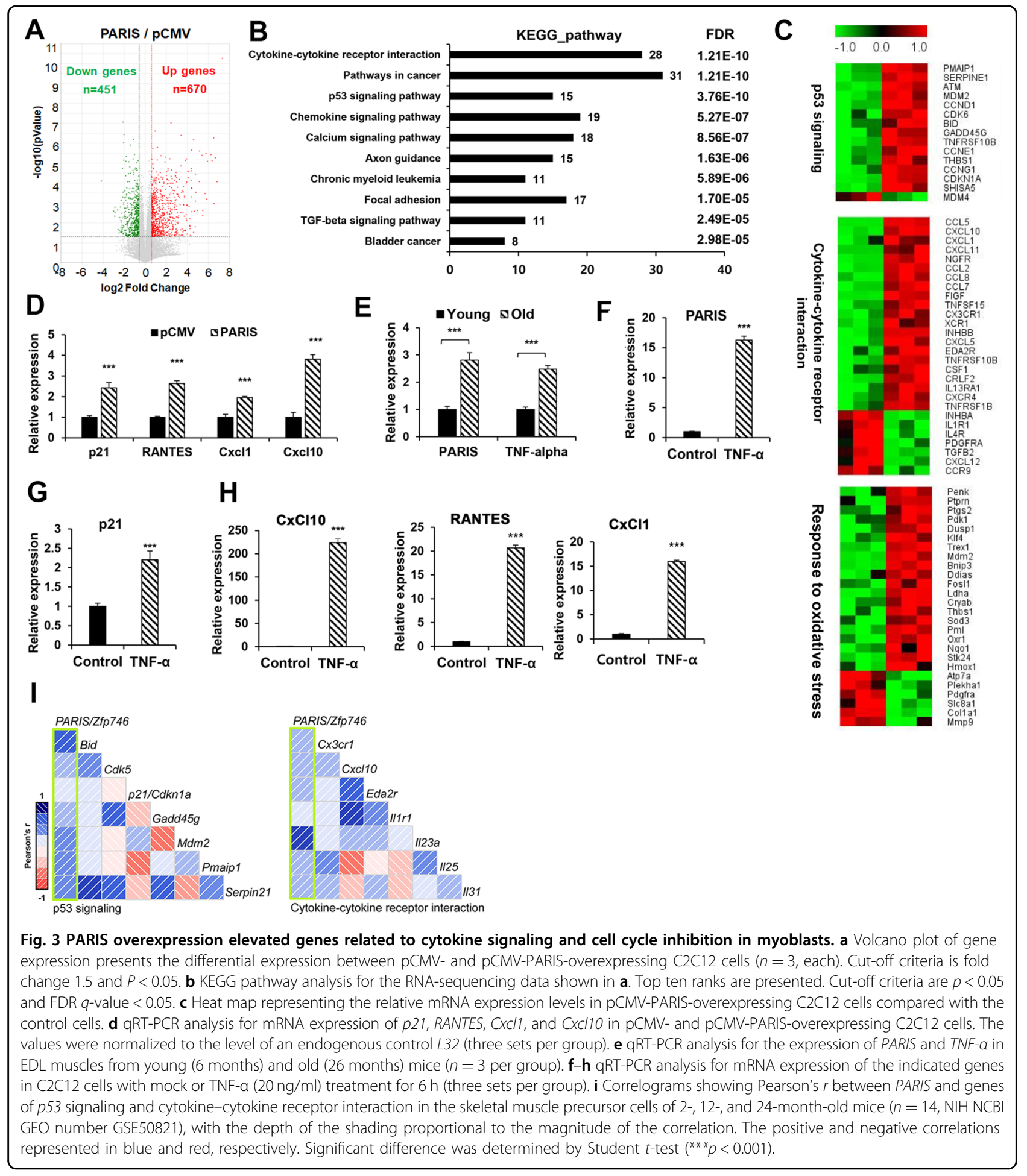

KEGG_p53 signaling (15 genes), KEGG_cytokinecytokine receptor interaction (28 genes), and GO_response to oxidative stress (26 genes) were significantly altered as depicted in a heat map (Fig. 3b, c). Among genes related to p53 signaling, p21 was increased about 1.6-fold. Furthermore, the reactome analysis revealed
PARIS-dependent alteration in genes related to G1 damage response signaling (Supplementary Fig. 6), likely reflecting the cellular senescence. The quantitative reverse-transcriptase PCR (qRT-PCR) analysis confirmed that PARIS-overexpressing myoblasts expressed higher levels of p21 ( 2.5-fold) and cytokines such as RANTES 


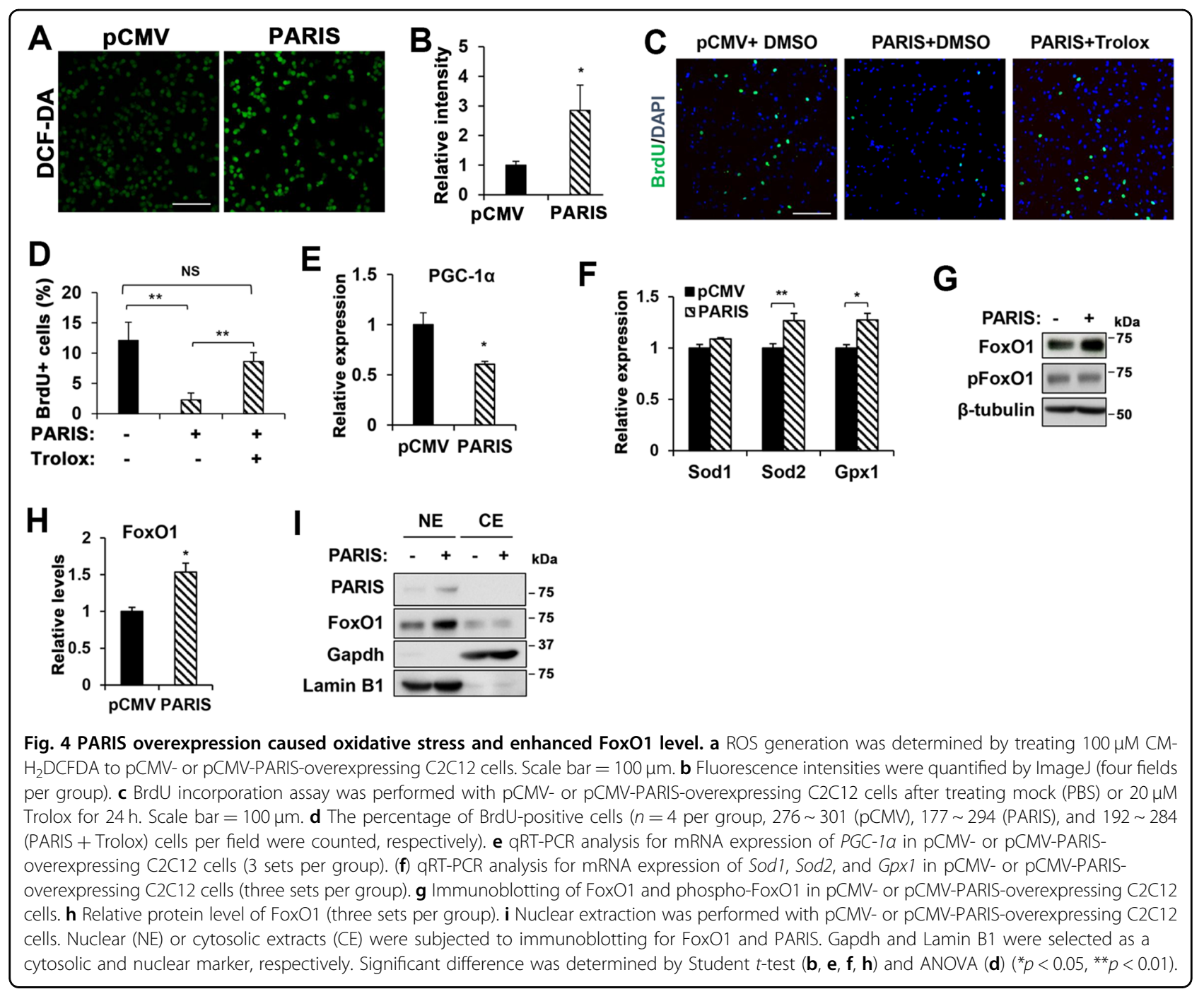

(Ccl5), Cxcl1, and Cxcl10 ( 2.5-4-fold) (Fig. 3d). These data suggest that PARIS overexpression causes alteration in inflammatory responses. Pro-inflammatory cytokines such as tumor necrosis factor (TNF)- $\alpha$ have been implicated in muscle atrophy related to cancer cachexia or aging $^{21,22}$. Consistently, TNF- $\alpha$ levels were significantly elevated in Extensor Digitorum Longus (EDL) muscles from 26-month-old (old) mice compared with 6-monthold (young) control muscles (Fig. 3e). PARIS expression was also greatly increased in old muscles, compared with the young muscles. This correlation made us to further examine the relationship between TNF- $\alpha$ and PARIS in myoblasts. C2C12 cells were treated with TNF- $\alpha$ (20 ng/ $\mathrm{ml}$ ) for $24 \mathrm{~h}$ and the expression of PARIS, p21, and other cytokines was assessed (Fig. 3f-h). TNF- $\alpha$ treatment greatly enhanced the level of PARIS about 16-fold and p21 about 2.3-fold. The expression of RANTES, Cxcl1, and Cxcl10 was greatly increased in TNF- $\alpha$-treated cells compared with the control cells. The close examination of previously published transcriptomes of the skeletal muscle progenitor cells of 2-, 12-, and 24-month-old mice $(n=14$, NIH NCBI GEO number GSE50821) revealed a positive correlation between PARIS, genes of p53 signaling, and cytokine-cytokine receptor interaction, similar to our current analysis in PARIS-overexpressing myoblasts (Fig. 3i). Taken together, PARIS overexpression causes alterations in inflammatory responses, response to oxidative stress, and p53 signaling related to cellular senescence.

\section{PARIS triggers oxidative stress response and FoxO1 accumulation in myoblasts}

As oxidative stress is one of the major trigger for cellular senescence, we next examined the effect of PARIS overexpression on oxidative stress in myoblasts. Control and PARIS-overexpressing $\mathrm{C} 2 \mathrm{C} 12$ cells were subjected to staining with 2',7'-dichlorofluorescin diacetate (DCF-DA) to detect the cellular reactive oxygen species (ROS). 
Unlike the control cells, PARIS-overexpressing cells showed a robust staining for DCF-DA with a threefold increase (Fig. 4a, b). To examine the role of oxidative stress in PARIS-triggered cell cycle arrest, control and PARIS-overexpressing myoblasts were treated with an antioxidant 6-hydroxy-2,5,7,8-tetramethylchroman-2carboxylic acid (Trolox, $20 \mu \mathrm{M}$ ) for $24 \mathrm{~h}$ followed by BrdU incorporation for $10 \mathrm{~min}$ and immunostaining. As previously shown, vehicle dimethyl sulfoxide-treated PARIS-overexpressing myoblasts exhibited greatly reduced BrdU incorporation, whereas Trolox treatment significantly restored the proliferation of PARISoverexpressing cells almost to the level of control cells (Fig. 4c, d). Thus, oxidative stress might be the cause for the impaired proliferation of PARIS-overexpressing myoblasts. Previous studies have shown that PARIS acts as a repressor for PGC- $1 \alpha$, which can be induced by oxidative stress and regulates the induction of antioxidant response genes ${ }^{17,23}$. It is conceivable that PARISmediated PGC- $1 \alpha$ repression might reduce the expression of antioxidant genes, such as Superoxide dismutase 1 (SOD1), SOD2, and Glutathione peroxidase $(G P X)^{23-26}$, contributing to cellular senescence. However, the qRTPCR analysis revealed that PGC-1 $\alpha$ expression was significantly reduced in PARIS-overexpressing myoblasts, whereas the expression of SOD1, SOD2, and GPX1 was mildly increased compared with the control levels in response to oxidative stress (Fig. 4e, f). Thus, it is concluded that the suppression of these antioxidant genes is not the major target of PARIS.

FoxO transcription factors are activated by multiple stresses including oxidative stress and the dysregulation of FoxOs has been implicated in cellular senescence through induction of $\mathrm{p} 21$ and $\mathrm{p} 27^{15,27}$. Considering the fact that PARIS and FoxOs can modulate gene expression through IRE, we have examined the involvement of FoxOs in PARIS-induced cellular senescence. PARISoverexpressing cells expressed higher levels of FoxO1 proteins, relative to control cells while the phosphorylated form of FoxO1 was unchanged (Fig. 4g, h), suggesting that the growth factor signaling-mediating FoxO1 inhibition occurs normally in PARISoverexpressing cells. The phosphorylation of FoxO1 induces its cytoplasmic localization thereby inhibiting its transcriptional activity ${ }^{12,13}$. Consistently, the level of cytoplasmic FoxO1 was unchanged in PARISoverexpressing myoblasts, whereas nuclear FoxO1 levels were elevated (Fig. 4i). As PARIS overexpression did not enhance FoxO1 mRNA levels in myoblasts (Supplementary Fig. 7a), it is concluded that PARIS overexpression elevates FoxO1 protein stability rather than the expression of FoxO1. Taken together, PARIS overexpression causes excessive oxidative stress leading to FoxO1 protein accumulation.

\section{Depletion of FoxO1 attenuates PARIS-induced senescence in myoblasts}

Next, the effect of FoxO1 overexpression on p21 expression was examined in $\mathrm{C} 2 \mathrm{C} 12$ myoblasts. In consistent with the previous report ${ }^{15,27,28}$, FoxO1 overexpression upregulated p21 levels in $\mathrm{C} 2 \mathrm{C} 12$ myoblasts, without elevating PARIS levels (Fig. 5a, b). Perturbation in autophagy is also implicated in cellular stress leading to cell death or senescence ${ }^{5,29-31}$. Thus, we have examined the autophagy response in control and PARISoverexpressing cells. Control and PARIS-overexpressing cells were cultured in normal growth medium or $0.1 \%$ fetal bovine serum (FBS) containing medium for $24 \mathrm{~h}$ and assessed autophagy by the level of lipidated LC3 (LC3-II) and p62. PARIS overexpression did not alter greatly autophagy (Supplementary Fig. 7b). In addition, we have examined the expression of autophagy-related genes, Atrogin1, LC3, Beclin, Atg5, Ulk1, and Bnip3 in cells with either PARIS overexpression or depletion (Supplementary Fig. 7c, d). The expression of autophagy-related genes did not alter significantly in relation with different PARIS levels. These data suggest that FoxO1 activation in response to PARIS does not alter autophagy. It can be further hypothesized that PARIS regulates p21 levels through FoxO1 leading to myoblast senescence. To test this hypothesis, control or PARIS-overexpressing $\mathrm{C} 2 \mathrm{C} 12$ cells were transfected with scrambled or FoxO1 siRNAs. Three different siFoxO1 were tested and siFoxO1-2 was used in further study due to efficient knockdown (Supplementary Fig. 8). FoxO1 depletion reduced the expression of p21 compared with the control cells (Fig. 5c-f). In consistent with the previous data, PARIS overexpression led to upregulation of FoxO1 and p21 protein and FoxO1 depletion in PARIS-overexpressing cells reduced both $\mathrm{p} 21$ mRNA and protein levels. Unlike the complete abrogation of p21 mRNA induction, p21 protein levels were less efficiently suppressed by FoxO1 depletion suggesting for a posttranslational mechanism to modulate $\mathrm{p} 21$ proteins in PARIS-overexpressing myoblasts. The effect of FoxO1 depletion on proliferation of PARIS-overexpressing cells was further examined by immunostaining for Ki67 and SA- $\beta$-Gal staining (Fig. 5g-i). The decrease in Ki67positive cell proportion in PARIS-overexpressing cells was partially but significantly attenuated by FoxO1 depletion. In contrast, the increase in SA- $\beta$-Gal-positive cell proportion was significantly decreased by FoxO1 depletion. Taken together, PARIS overexpression augments excessive oxidative stress and FoxO1 activity, contributing to cellular senescence in myoblasts.

\section{PARIS enhances the recruitment of FoxO1 to the $p 21$ promoter region}

As PARIS overexpression resulted in nuclear FoxO1 accumulation, we have assessed the recruitment of FoxO1 


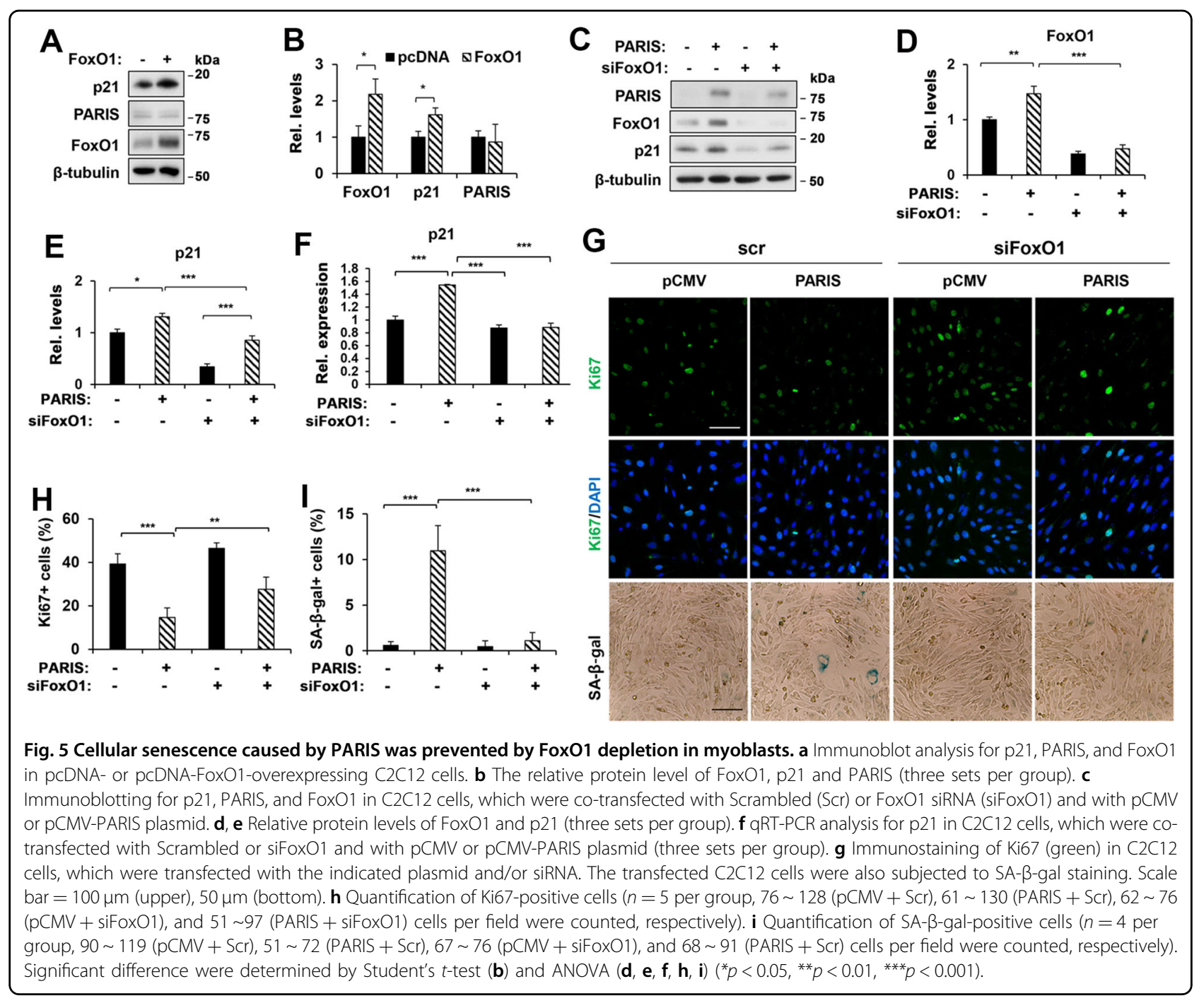

and PARIS in three IRE-containing regions $(-888 /-620$; $-1524 /-1253 ;-2789 /-2446)$ of the upstream regulatory region in the $p 21$ promoter (Fig. 6a). Interestingly, the chromatin immunoprecipitation (ChIP) assay revealed the specific recruitment of FoxO1 to the region -1524/-1253 and the PARIS overexpression elicited a robust enrichment of FoxO1 to this region. In consistent with the rescuing effects of Trolox on cell proliferation shown in Fig. 4c, the FoxO1 enrichment to this promoter region was blunted by Trolox treatment (Fig. 6b). Unfortunately, we were unable to immunoprecipitate with the commercially available PARIS antibodies tested in this study. Thus we have used the anti-Flag antibody to precipitate the overexpressed Flag-tagged PARIS on chromatin (Fig. $6 \mathrm{~b})$. The ChIP experiment with anti-Flag antibody showed a specific enrichment of PARIS in the same region of the $p 21$ promoter like FoxO1, suggesting that PARIS might be co-recruited with FoxO1. Unlike FoxO1 recruitment to the $p 21$ promoter, PARIS recruitment was not affected by the Trolox treatment. These data suggest that the FoxO1 recruitment to the $\mathrm{p} 21$ regulatory region is triggered by oxidative stress. We then have examined the potential physical interaction between PARIS and FoxO1 in 293T cells (Fig. 6c). PARIS and FoxO1 were reciprocally co-immunoprecipitated when overexpressed. Based on the effect of TNF- $\alpha$ on PARIS and p21 upregulation, $\mathrm{C} 2 \mathrm{C} 12$ cells were treated with TNF- $\alpha$ and analyzed for the interaction between PARIS and FoxO1 (Fig. 6d). The treatment of TNF- $\alpha$ elevated the interaction between FoxO1 and PARIS, likely contributing to $\mathrm{p} 21$ upregulation (Fig. 6e, f). To assess the effect of PARIS depletion on FoxO1 recruitment to $p 21$ promoter region, control or siPARIS-expressing $\mathrm{C} 2 \mathrm{C} 12$ cells were subjected to ChIP assay. FoxO1 recruitment was significantly reduced by PARIS depletion (Fig. 6g). Next the effect of FoxO1 depletion on PARIS recruitment to $p 21$ promoter was examined. The depletion of FoxO1 did not affect the enrichment of PARIS to $p 21$ promoter, whereas the 


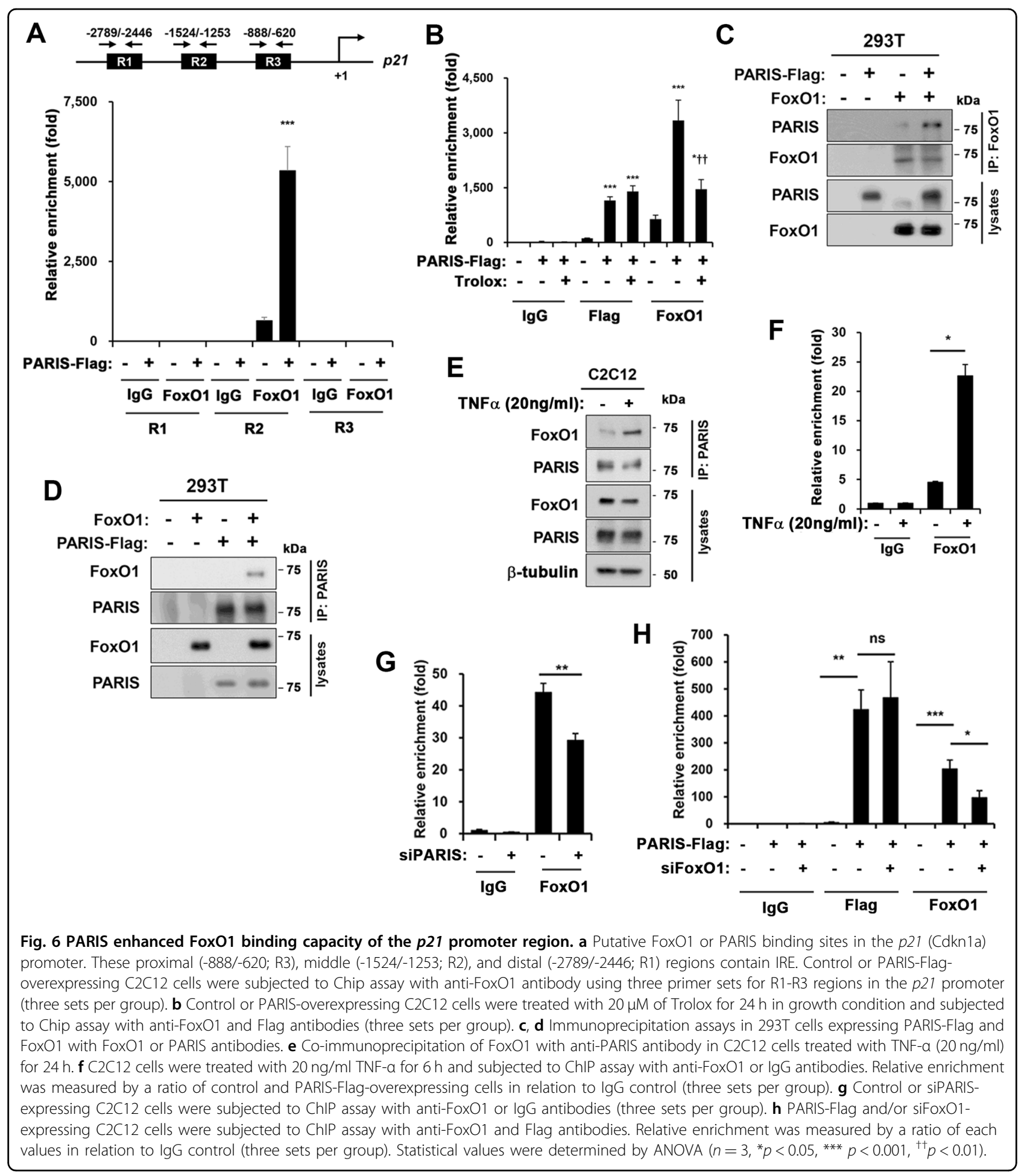

PARIS-mediated FoxO1 recruitment was significantly reduced by FoxO1 depletion (Fig. 6h). Taken together, these data suggest that PARIS and FoxO1 are co-recruited to $p 21$ promoter in response to oxidative stress.

In addition to PARIS/FoxO1/p21 axis, the global gene expression profile suggested the potential involvement of p53 signaling in PARIS-mediated p21 expression. As overexpression in myoblasts elevated p53 proteins (Fig. $2 \mathrm{j}$ ), there was no change in p53 mRNA levels observed from the RNA-sequencing data (Fig. 4a). PARIS overexpression appears to regulate 553 protein accumulation, likely related to oxidative stress observed in PARIS-overexpressing 
myoblasts. Thus, we examined the effect of p53 depletion on p21 expression in response to PARIS overexpression. $\mathrm{C} 2 \mathrm{C} 12$ cells were transfected with scrambled or three different p53 siRNAs (Supplementary Fig. 9a). Among the three different sip53, sip53\#3 was used in further study. The p21 expression was significantly decreased in both control and PARIS-overexpressing myoblasts (Supplementary Fig. 9b, c). In conclusion, these data suggest that PARIS induces p21 expression through p53 and FoxO1 leading to impaired cell proliferation and cellular senescence.

\section{Discussion}

Oxidative stress in muscle stem cells has been linked with decreased regenerative capacity accompanied by cell death or senescence ${ }^{5,32}$. In this study, we demonstrate that PARIS overexpression induced cellular senescence with elevated DNA damage accompanied by impaired proliferation and differentiation of myoblasts. Interestingly, PARIS overexpression elevated ROS levels and Trolox treatment restored proliferation of PARIS-overexpressing cells, suggesting that PARIS overexpression perturbs the control mechanism of redox homeostasis. Previously PARIS has been associated with Parkinson's diseases ${ }^{17}$. PARIS is targeted by Parkin to be degraded and in Parkinson's disease the level of PARIS increases leading to repression of PGC- $1 \alpha$ and mitochondrial dysfunction ${ }^{17,33}$. PGC-1 $\alpha$ levels are critical for the expression of antioxidant genes, such as $S O D 1, S O D 2$, and GPx $1^{26}$. PGC$1 \alpha$-deficient fibroblasts and mice are more sensitive to oxidative stress $^{34}$. PARIS-overexpressing myoblasts exhibited decreased PGC-1 $\alpha$ transcript however the levels of SOD1, SOD2, and GPx1 genes were rather slightly increased. Thus, it can be concluded that the induction of antioxidant gene expression by PGC- $1 \alpha$ might not be the major defect in PARIS-overexpressing cells. However, we cannot entirely rule out the partial contribution of PGC$1 \alpha$ in PARIS-elicited cellular senescence.

FoxOs have been implicated in the control of diverse cellular processes, including autophagy, stem cell quiescence, and senescence ${ }^{10,12}$. FoxO1 activates the expression of cell cycle inhibitors, p21 or p27, through interaction to FoxO-response element in the promoter region of these genes ${ }^{15,27,35}$. PARIS overexpression upregulated the levels of p21 and p27 proteins likely contributing to cell cycle inhibition and senescent phenotype of myoblasts. FoxO1 and p53 hyperactivation seems to be linked with p21 induction and cellular senescence of PARIS-overexpressing myoblasts. PARIS-overexpressing cells expressed higher levels of FoxO1 and p53 proteins without upregulated transcription of these genes. Similar to PARIS overexpression, FoxO1 overexpression increased p21 levels. However, PARIS levels were unchanged by FoxO1 overexpression, suggesting that
FoxO1 might be acting downstream of PARIS. In consistent with this assumption, FoxO1 depletion in PARISoverexpressing cells reduced p21 levels and restored myoblast proliferation. In addition, FoxO1 depletion in PARIS-overexpressing cells reduced DNA damage response and senescent phenotypes. Thus, these data suggest that the increased PARIS levels might perturb the redox homeostasis through yet to be identified mechanism and this will elevate FoxO1 and p53 levels contributing to cell cycle inhibition and senescence.

PARIS seems to regulate FoxO1 protein stability or nuclear localization. However, the exact mechanism is currently unclear. As the level of phosphorylated FoxO1 did not change in PARIS-overexpressing cells, it appears that PARIS does not interfere with AKT-mediated FoxO1 phosphorylation leading to cytosolic localization and degradation of FoxO $1^{12,28}$. One possible mechanism might be through a physical interaction and corecruitment of PARIS and FoxO1 to the $p 21$ promoter region between -1253 and -1524 . Furthermore, PARIS overexpression enhanced the recruitment of FoxO1 to this region, which was attenuated by the Trolox treatment. Unlike FoxO1, the Trolox treatment did not affect the level of PARIS recruited to the $p 21$ promoter region. Thus, it can be concluded that FoxO1 recruitment to the $p 21$ promoter region is dependent on cellular oxidative stress. Recently, FoxO1 has been shown to induce cellular senescence in ovarian cancer cells by the co-recruitment with progesterone receptor to the $p 21$ promoter region $^{36}$. Similarly, FoxO1 appears to be co-recruited with PARIS in the $p 21$ promoter region containing IRE. Due to the technical difficulty, our data are based on the PARIS overexpression study. Thus, we need to verify the recruitment of endogenous PARIS under oxidative stress condition, which might provide more accurate physiological role of PARIS in cell senescence in response to oxidative stress.

Although the endogenous signaling controlling PARIS expression is currently unclear, PARIS levels were greatly elevated along with TNF- $\alpha$ in aged muscles, relative to young muscles. Interestingly, inflammatory cytokines, such as TNF- $\alpha$ evoke generation of ROS and elevated inflammatory signals have been closely linked with muscle aging and decreased regenerative capacity ${ }^{21,22}$. Thus, it is well likely that PARIS is involved in oxidative stress related to muscle aging through corroborating with TNF$\alpha$. In support of this hypothesis, the expression of PARIS and p21 was augmented by TNF- $\alpha$ treatment in myoblasts. Further studies will be required to elucidate the role of PARIS in TNF- $\alpha$ mediated inflammatory responses and muscle stem cell dysfunction. Our current study demonstrates a suppressive role of PARIS in muscle regeneration through perturbation of redox homeostasis. Similar to the strategy proposed to treat Parkinson's 
diseases, PARIS upregulation needs to be prevented to maintain muscle regenerative capacity. Consistent with this notion, Parkin overexpression has been shown to prevent muscle atrophy related to muscle aging ${ }^{37}$. Considering the fact that Parkin can suppress PARIS, the effects observed by Parkin overexpression could be at least partly contributed by PARIS reduction. Thus, PARIS might represent an attractive target to prevent agingrelated diseases, such as Parkinson's diseases as well as sarcopenia.

\section{Materials and methods}

\section{Cell culture, counting, and transfection}

C2C12 myoblasts, 293T cells, and MEF cultures were carried out as previously described ${ }^{38,39}$. C2C12 cells were cultured in Dulbecco modified Eagle medium (DMEM) containing 15\% FBS, and $1 \times$ Penicillin-Streptomycin. To induce myogenic differentiation, cells were cultured in DMEM containing $2 \%$ horse serum at near confluence. MEFs were isolated from E13.5 embryos of C57BL/6 mice and cultured in DMEM containing 10\% FBS and $1 \times$ Penicillin-Streptomycin. 10T1/2 cells were cultured in DMEM containing 10\% FBS and $1 \times$ Penicillin-Streptomycin. For passaging MEFs, $\sim 3 \times 10^{5}$ cells were plated and counted 2 days later, to determine the growth rate. The numbers of viable cells, which were excluded from staining with Trypan blue were counted by using hemocytometer. For transfection, pCMV, pCMVFlag-PARIS, pcDNA3, pcDNA3-FoxO1, or siRNAs were transfected to the corresponding cells with Lipofectamin 2000 (Invitrogen), according to manufacturer's instructions. siRNA sequences are listed in Supplementary Table 1. Recombinant human TNF- $\alpha$ and Trolox were purchased from Abcam and Sigma-Aldrich, respectively.

\section{Immunostaining, immunoblotting, and immunoprecipitation}

Immunocytochemisty were performed as previously described $^{39,40}$. The fixed cells were incubated with primary antibodies including anti-PARIS (Millipore, MABN476), anti-PARIS (Abcam, ab130867), anti-MHC (DSHB, MF20), anti-Ki67 (Abcam, ab15580), anti-p21 (Santa Cruz, sc-471), anti-phospho- $\gamma \mathrm{H} 2 \mathrm{AX}$ (Ser139) (Cell Signaling, 9718), anti-Biotin (Jakson, 115-065-146), and anti-Flag (Antibody Frontier, LF-PA20169) antibody. Then, incubation with Alexa Fluor 488 goat anti-mouse antibody or Alexa Fluor 555 goat anti-rabbit antibody (Invitrogen) was performed for $1 \mathrm{~h}$ at room temperature. Images were captured by Zeiss LSM-510 Meta confocal microscope and Nikon ECLIPSE TE-2000U microscope. NIS-Elements F software (Nikon) and ImageJ software were employed to quantify for images. To evaluate ROS production in cells, cells were treated with $100 \mu \mathrm{M}$ CM$\mathrm{H}_{2}$ DCFDA (Invitrogen) for $30 \mathrm{~min}$. Fluorescence images in live cells were captured using Zeiss LSM-510 Meta confocal microscope and quantified by ImageJ.

Immunoblot analysis were performed as described previously ${ }^{40}$. Cell lysates were prepared by RIPA buffer (Invitrogen) and separated by SDS-polyacrylamide gel electrophoresis (PAGE). The blots were incubated with primary antibodies including anti-PARIS (Millipore, MABN476), anti-Myogenin (DSHB, F5D), anti-MHC (DSHB, MF20), anti-Caspase3 (Santa Cruz, sc-7148), anti-p21 (Santa Cruz, sc-471), anti-p27 (Abcam, ab7961), anti-p53 (Santa Cruz, sc-6243), anti-FoxO1 (Cell Signaling, 2880S), anti-phospho-FoxO1 (Ser256) (Cell Signaling, 9461S), anti-GAPDH (Antibody Frontier, LF-PA0018), anti-Lamin B1 (Abcam, ab16048), anti-PGC-1 $\alpha$ (Calbiochem, ST1202), TnT (Abcam, ab10214), anti-MyoD (Santa Cruz, sc-760), anti-LC3 (Sigma-Aldrich, L8918), anti-p62 (Santa Cruz, sc-48402), and anti- $\beta$-tubulin antibody (Sigma-Aldrich, T5293). Then, samples were incubated with appropriate secondary antibodies conjugated with horseradish peroxidase and signals were detected by ECL (West Save).

Immunoprecipitation assays were carried out as previously described ${ }^{41}$. Cells for co-immunoprecipitation were lysed by extraction buffer containing $10 \mathrm{mM}$ Tris$\mathrm{HCl}$ (pH 7.4), $150 \mathrm{mM} \mathrm{NaCl}, 1 \mathrm{mM}$ EDTA, 1\% Triton X100 and complete protease inhibitor cocktail (Roche). Then, samples were incubated with magnetic beads (Invitrogen) and anti-FoxO1 antibody at $4^{\circ} \mathrm{C}$ overnight. Samples were boiled and separated by SDS-PAGE. Subsequently, dissociated proteins were detection by antiPARIS antibody.

\section{Quantitative RT-PCR}

Total RNA from cells were isolated with Easy Blue (Intron), according to manufacturer's instructions. After that, cDNA was synthesized using PrimeScript ${ }^{\mathrm{TM}}$ cDNA synthesis kit (TaKaRa) according to the manufacturer's instructions. qRT-PCR were performed by real-time PCR system with SYBR Premix ExTag ${ }^{\mathrm{TM}}$ according to the manufacturer's instructions (TaKaRa). Primer sequences are listed in Supplementary Table 2.

\section{Library preparation and RNA sequencing}

For RNA sequencing, transfected confluent $\mathrm{C} 2 \mathrm{C} 12$ cells were incubated in the medium containing $0.1 \%$ FBS for $24 \mathrm{~h}$. Transcriptional profiles were analyzed by e-Biogen corporation. Libraries were prepared from total RNA using the SMARTer Stranded RNA-Seq Kit (Clontech Laboratories). The isolation of mRNA was performed using the Poly(A) RNA Selection Kit (LEXOGEN). The isolated mRNAs were used for the cDNA synthesis and shearing, following manufacture's instruction. Indexing was performed using the Illumina indexes 1-12. The enrichment step was carried out using of PCR. 
Subsequently, libraries were checked using the Agilent 2100 bioanalyzer (DNA High Sensitivity Kit) to evaluate the mean fragment size. Quantification was performed using the library quantification kit using a StepOne RealTime PCR System (Life Technologies). Highthroughput sequencing was performed as paired-end 100 sequencing using HiSeq 2500 (Illumina). For data analysis, mRNA-Seq reads were mapped using TopHat software tool in order to obtain the alignment file. Differentially expressed genes were determined based on counts from unique and multiple alignments using coverage in Bedtools. The RT (Read Count) data were processed based on Quantile normalization method using EdgeR within R (R development Core Team, 2016) using Bioconductor. The alignment files also were used for assembling transcripts, estimating their abundances and detecting differential expression of genes or isoforms using cufflinks. And we used the FPKM (fragments per kilobase of exon per million fragments) as the method of determining the expression level of the gene regions.

Transcriptomes of the skeletal muscle precursor cells of 2-, 12-, and 24-month-old mice were obtained from the publicly available data of the NIH NCBI Gene Expression Omnibus ( $n=14$, NIH NCBI GEO number GSE50821). Correlogram was generated using RStudio (R Consortium Inc, Boston, MA) as described in the previous study ${ }^{42}$.

\section{BrdU incorporation, cell apoptosis, and cellular senescence assay}

For BrdU incorporation, $10 \mu \mathrm{M}$ BrdU (Sigma-Aldrich, B5002) was treated to the cells in the dark for 10 15 min. The treated cells were fixed and permeabilized with $2 \mathrm{~N}$ $\mathrm{HCl}$ at 37 degrees for $30 \mathrm{~min}$. After neutralizing with $0.1 \mathrm{M}$ borate buffer ( $\mathrm{pH} \mathrm{8.5)}$ for $12 \mathrm{~min}$, cells were incubated with anti-BrdU antibody (Chemicon, MAB3222) at $4{ }^{\circ} \mathrm{C}$ during overnight. Then, cells were incubated with Alexa Fluor 488 goat anti-mouse antibody at room temperature for $1 \mathrm{~h}$. Images were captured by Zeiss LSM510 Meta confocal microscope and Nikon ECLIPSE TE2000 U microscope. NIS-Elements F software (Nikon) and ImageJ software were employed to quantify for images.

Apoptosis analysis was performed by staining with Annexin-V-FITC dye (Bio bud), according to manufacturer's protocols. Dissociated $\mathrm{C} 2 \mathrm{C} 12$ cells were incubated with Annexin-V-FITC dye for $15 \mathrm{~min}$ in cold PBS and then these cells were resuspended in fluorescenceactivated cell sorting (FACS) buffer. Detection of Annexin-V-FITC $(518 \mathrm{~nm})$ and PI $(620 \mathrm{~nm})$ was performed by FACS (FACS Canto2), along with $\mathrm{H}_{2} \mathrm{O}_{2}$-treated positive and not stained negative controls.

To assess cellular senescence, SA- $\beta$-Gal assay was carried out by using SA- $\beta$-Gal assay kit (Cell signaling), according to manufacturer's protocols. Briefly, fixed cells were stained with the provided $\mathrm{pH} 5.9 \sim 6.1$ mixture containing $\beta$-gal overnight. GL13 (Sentragor) staining were performed by manufacturer's protocols. Images of SA- $\beta$-Gal and GL13-positive cells were captured by Nikon ECLIPSE TE-2000U microscope.

\section{Cytoplasmic/nuclear fraction}

Cells were incubated with cytoplasm extracts buffer containing $10 \mathrm{mM}$ HEPES (pH 7.9), 0.08\% Triton X-100, $50 \mathrm{mM} \mathrm{NaCl}, 0.5 \mathrm{M}$ Sucrose, $0.1 \mathrm{mM}$ EDTA, and proteinase inhibitor cocktail (Roche) on ice for $5 \mathrm{~min}$ and after centrifugation (2000 r.p.m. for $15 \mathrm{~min}$ ), the supernatant was taken for cytoplasmic fraction. Following washing the pellet with Wash buffer (10 mM HEPES (pH 7.9), $10 \mathrm{mM}$ $\mathrm{KCl}, 0.1 \mathrm{mM}$ EDTA and proteinase inhibitor cocktail) twice, the pellet was lysed with Nuclear extract buffer containing $10 \mathrm{mM}$ HEPES (pH 7.9), $500 \mathrm{mM} \mathrm{NaCl}$, $0.1 \mathrm{mM}$ EDTA, $0.1 \% \mathrm{NP} 40$, and proteinase inhibitor cocktail on ice for $30 \mathrm{~min}$ and the supernatant was taken for nuclear fraction.

\section{Chromatin immunoprecipitation assay}

ChIP analysis was carried out as previously described. Antibodies used in this study are as following; rabbit IgG (Millipore, 12-370), FoxO1 (Cell signaling, 2880 S) and Flag (Sigma-Aldrich, F3040). The primers used to amplify the $p 21$ promoter regions designated as $\mathrm{R} 1(-620 /-888)$, R2 (-1253/-1524), and R3 (-2446/-2789) are listed in Supplementary Table 2.

\section{Statistical analysis}

Statistical differences between two or multiple groups were analyzed by paired or unpaired two-tailed Student's $t$-test or one-way analysis of variance test. All results are representative of three or more independent experimental sets. Data are expressed as means \pm SD or \pm SEM, as indicated in the figure legends. Differences were considered statistically significant as ${ }^{*} p<0.05$, ${ }^{* *} p<0.01$, and $\approx * * 0.001$.

\section{Acknowledgements}

This research was supported by the National Research Foundation Grant funded by the Korean Government (MSIP) (NRF-2019R1A2C2006233; NRF2017M3A9D8048710; NRF-2016R1A5A2945889).

\section{Author details \\ ${ }^{1}$ Department of Molecular Cell Biology, Sungkyunkwan University School of Medicine, Suwon 440-746, Republic of Korea. ${ }^{2}$ Single Cell Network Research Center, Sungkyunkwan University School of Medicine, Suwon 440-746, Republic of Korea. ${ }^{3}$ Well Aging Research Center, Daegu Gyeongbuk Institute of Science and Technology (DGIST), Daegu 42988, Republic of Korea}

\section{Data availability}

All relevant data are included in the main article and Supplementary Figures. Additional information that support the findings of this study are available upon request from the corresponding author.

Conflict of interest

The authors declare that they have no conflict of interest. 


\section{Publisher's note}

Springer Nature remains neutral with regard to jurisdictional claims in published maps and institutional affiliations.

Supplementary Information accompanies this paper at (https://doi.org/ 10.1038/s41419-020-2552-7).

Received: 16 September 2019 Revised: 6 February 2020 Accepted: 9 March 2020

Published online: 12 May 2020

\section{References}

1. Church, J. C. T., Noronha, R. F. X. \& Allbrook, D. B. Satellite cells and skeletal muscle regeneration. Br. J. Surg. 53, 638-642 (1966).

2. Järvinen, T. A., Järvinen, T. L., Kääriäinen, M., Kalimo, H. \& Järvinen, M. Muscle injuries: biology and treatment. Am. J. Sports Med. 33, 745-764 (2005).

3. Schiaffino, S., Dyar, K. A., Ciciliot, S., Blaauw, B. \& Sandri, M. Mechanisms regulating skeletal muscle growth and atrophy. FEBS J. 280, 4294-4314 (2013).

4. Rando, T. A. Stem cells, ageing and the quest for immortality. Nature $\mathbf{4 4 1}$ 1080-1086 (2006).

5. García-Prat, L. et al. Autophagy maintains stemness by preventing senescence. Nature 529, 37-42 (2016).

6. Sousa-Victor, P. et al. Geriatric muscle stem cells switch reversible quiescence into senescence. Nature 506, 316-321 (2014).

7. Sousa-Victor, P., García-Prat, L. \& Munoz-Canoves, P. New mechanisms driving muscle stem cell regenerative decline with aging. Int. J. Dev. Biol. 62, 583-590 (2018).

8. Blanc, R. S., Vogel, G., Chen, T., Crist, C. \& Richard, S. PRMT7 preserves satellite cell regenerative capacity. Cell Rep. 14, 1528-1539 (2016).

9. Latorre, E., Ostler, E. L., Faragher, R. G. \& Harries, L. W. FOXO1 and ETV6 genes may represent novel regulators of splicing factor expression in cellular senescence. FASEB J. 33, 1086-1109 (2018).

10. Muñoz-Espín, D. et al. Programmed cell senescence during mammalian embryonic development. Cell 155, 1104-1118 (2013).

11. Yamashita, A., Hatazawa, Y., Hirose, Y., Ono, Y. \& Kamei, Y. FOXO1 delays skeletal muscle regeneration and suppresses myoblast proliferation. Biosci. Biotechnol. Biochem. 80, 1531-1535 (2016).

12. Calnan, D. \& Brunet, A. The foxo code. Oncogene 27, 2276-2288 (2008).

13. Storz, P. Forkhead homeobox type $O$ transcription factors in the responses to oxidative stress. Antioxid. Redox Signal. 14, 593-605 (2011).

14. Modur, V., Nagarajan, R., Evers, B. M. \& Milbrandt, J. FOXO proteins regulate tumor necrosis factor-related apoptosis inducing ligand expression Implications for PTEN mutation in prostate cancer. J. Biol. Chem. 277, 47928-47937 (2002).

15. Seoane, J., Le, H.-V., Shen, L., Anderson, S. A. \& Massagué, J. Integration of Smad and forkhead pathways in the control of neuroepithelial and glioblastoma cell proliferation. Cell 117, 211-223 (2004).

16. Vezzali, R. et al. The FOXG1/FOXO/SMAD network balances proliferation and differentiation of cortical progenitors and activates Kcnh3 expression in mature neurons. Oncotarget 7, 37436-37455 (2016).

17. Shin, J.-H., Ko, H. S., Kang, H., Lee, Y. \& Lee, Y.-I. et al. PARIS (ZNF746) repression of PGC-1a contributes to neurodegeneration in Parkinson's disease. Cell 144, 689-702 (2011).

18. Fernandez-Marcos, P. J. \& Auwerx, J. Regulation of PGC-1a, a nodal regulator of mitochondrial biogenesis. Am. J. Clin. Nutr. 93, 884S-890S (2011).

19. Jung, J. H. et al. Zinc finger protein 746 promotes colorectal cancer progression via c-Myc stability mediated by glycogen synthase kinase $3 \beta$ and Fbox and WD repeat domain-containing 7. Oncogene 37, 3715-3728 (2018).
20. Gorgoulis, V. et al. Cellular senescence: defining a path forward. Cell $\mathbf{1 7 9}$ 813-827 (2019).

21. Coletti, D., Moresi, V., Adamo, S., Molinaro, M. \& Sassoon, D. Tumor necrosis factor-a gene transfer induces cachexia and inhibits muscle regeneration. Genesis 43, 120-128 (2005).

22. Reid, M. B. \& Li, Y.-P. Tumor necrosis factor-a and muscle wasting: a cellular perspective. Respir. Res. 2, 269-272 (2001).

23. St-Pierre, J. et al. Suppression of reactive oxygen species and neurodegeneration by the PGC-1 transcriptional coactivators. Cell 127, 397-408 (2006).

24. Aquilano, K. et al. p53 orchestrates the PGC-1a-mediated antioxidant response upon mild redox and metabolic imbalance. Antioxid. Redox Signal. 18, 386-399 (2013).

25. Baldelli, S., Aquilano, K. \& Ciriolo, M. PGC-1a buffers ROS-mediated removal of mitochondria during myogenesis. Cell Death Dis. 5, e1515 (2014).

26. Zhang, T. et al. FoxO1 plays an important role in regulating $\beta$-cell compensation for insulin resistance in male mice. Endocrinology 157, 1055-1070 (2016).

27. Medema, R. H., Kops, G. J., Bos, J. L. \& Burgering, B. M. AFX-like Forkhead transcription factors mediate cell-cycle regulation by Ras and PKB through p27 kip1. Nature 404, 782-787 (2000).

28. Tia, N. et al. Role of Forkhead Box O (FOXO) transcription factor in aging and diseases. Gene 648, 97-105 (2018).

29. Han, X. et al. AMPK activation protects cells from oxidative stress-induced senescence via autophagic flux restoration and intracellular NAD+ elevation. Aging Cell 15, 416-427 (2016).

30. Mai, S., Muster, B., Bereiter-Hahn, J. \& Jendrach, M. Autophagy proteins LC3B, ATG5 and ATG12 participate in quality control after mitochondrial damage and influence lifespan. Autophagy 8, 47-62 (2012).

31. Tai, H. et al. Autophagy impairment with lysosomal and mitochondrial dysfunction is an important characteristic of oxidative stress-induced senescence. Autophagy 13, 99-113 (2017).

32. L'honoré, A. et al. The role of Pitx2 and Pitx3 in muscle stem cells gives new insights into P38a MAP kinase and redox regulation of muscle regeneration. Elife 7, e32991 (2018).

33. Stevens, D. A. et al. Parkin loss leads to PARIS-dependent declines in mitochondrial mass and respiration. Proc. Natl Acad. Sci. USA 112, 11696-11701 (2015).

34. Liu, C., Li, S., Liu, T., Borjigin, J. \& Lin, J. D. Transcriptional coactivator PGC-1a integrates the mammalian clock and energy metabolism. Nature 447 477-481 (2007).

35. Jiang, G. et al. Transcriptional and post-transcriptional upregulation of p27 mediates growth inhibition of isorhapontigenin (ISO) on human bladder cancer cells. Carcinogenesis 39, 482-492 (2018).

36. Diep, C. et al. Progesterone receptors induce FOXO1-dependent senescence in ovarian cancer cells. Cell Cycle 12, 1433-1449 (2013).

37. Leduc-Gaudet, J. P., Reynaud, O., Hussain, S. N. \& Gouspillou, G. Parkin overexpression protects from ageing-related loss of muscle mass and strength. J. Physiol. 597, 1975-1991 (2019).

38. Jeong, H.-J. et al. Prmt7 promotes myoblast differentiation via methylation of p38MAPK on arginine residue 70. Cell Death Differ. 27, 573-586 (2020).

39. Vuong, T. A. et al. PRMT7 methylates and suppresses GLI2 binding to SUFU thereby promoting its activation. Cell Death Differ. 27, 15-28 (2020).

40. Choi, S. et al. Skeletal muscle-specific Prmt1 deletion causes muscle atrophy via deregulation of the PRMT6-FOXO3 axis. Autophagy 15, 1069-1081 (2019).

41. Pyun, J.-H. et al. Cardiac specific PRMT1 ablation causes heart failure through CaMKIl dysregulation. Nat. Commun. 9, 5107 (2018).

42. Chung, H. K. et al. Growth differentiation factor 15 is a myomitokine governing systemic energy homeostasis. J. Cell Biol. 216, 149-165 (2017). 